\title{
Editorial
}

\section{Subtalar Dislocations, Total Talar Dislocations and the Extruded Talus: A Polynymous Injury or Separate Entities?}

The talus participates in the formation of three important joints: the ankle (talocrural joint), the subtalar joint and the talonavicular joint. Integrity of these joints is paramount for maintaining normal biomechanics of the foot. Due to the rarity of injuries to this bone, the average orthopod may confuse some of these injuries, and may have a limited understanding of the injuring mechanism in others. The literature speaks of 'talus dislocations' somewhat vaguely, an entity very different from talus fractures, using a significant degree of sloppiness, with publications discussing talus dislocations and extrusions somewhat unclearly. Is this then a case of a rare but complex injury being reffered to by different names by different authors? This maybe the unanswered question.

\section{Sanskrit avyākrta, Pali: avyākata—unfathomable, unexpounded.}

Even in Buddhism, there is a set of common philosophical questions that Buddha refused to answer, as the answers were confusing, and Buddha described them as 'a net and refused to be drawn into such a net of theories, speculations and dogmas'. Modern medicine, on the other hand, loves theories, using them to explain complex issues, which in the end need to be supported by facts and evidence. In the first phase of our explanation of talar dislocations, we propose a theory of these being a polynymous injury.

We did an internet search for the definition of a person with multiple names; the nearest that Wikipedia could describe it was with the term 'polynymous' meaning 'having multiple names'. Is that term applicable to talus dislocations or extrusions, with the injury mechanism being a continuum of the same process, but being aborted at different stages?

The talus can dislocate from one or more of its articulations with a specific mechanism of plantarflexion, associated with medial or lateral force. It is important to note that body dislocations associated with neck of talus fractures, with the body extruded posteriorly happen with a dorsiflexion injury and forward glide of the foot, which maybe the exact opposite. Dislocation of the talus from the subtalar and the talonavicular joints is rightly termed as 'subtalar' or 'peritalar' dislocation. It is important to understand that it is not the talus that dislocates per se, rather it is the foot distal to the talus that is displaced by the deforming forces, while the talus and ankle remain intact, albeit with a degree of plantarflexion. Subtalar dislocations maybe medial or lateral, depending on the displacement of the foot relative to the talus. Medial subtalar dislocations occur as a result of inversion and supination forces and are usually low-energy injuries. The calcaneus along with the navicular and the rest of the foot swivels medially around the sustentaculum tali with disruption of the talonavicular and talocalcaneal ligaments. Lateral subtalar dislocations, on the other hand, occur as a result of high-energy trauma; an eversion force displaces the foot laterally, with the anterior process of calcaneus acting as the fulcrum. Whereas medial subtalar dislocations are often thought to have an excellent outcome after reduction, lateral dislocations are often associated with other foot injuries and poorer outcomes thereof. ${ }^{1}$

A total talar dislocation, in contrast to a subtalar dislocation, involves dislocation of the talus from all of its three articulations. It is believed that at first forced plantarflexion causes the foot to dislocate as a whole from the ankle joint. Subsequently, continuing adduction and internal rotation forces cause the foot to rotate $90^{\circ}$ along its vertical and longitudinal axis, thereby dislocating the talus from the talocalcaneal and talonavicular articulations. Finally, the displacing foot returns to its position leaving the talus dislocated laterally. Similarly, eversion forces may lead to total talar dislocation with the talus lying medially. Both of these are high energy mechanisms. ${ }^{2}$

Talar extrusions are said to occur when the talus is dislocated from all of its articulations and is extruded through an open wound. The talus may protrude through the open wound and may have residual ligamentous connections; this is termed as a partial talar extrusion. When the talus is completely extruded from the foot and lies outside the body, often with no ligamentous connections, it is termed as total talar extrusion or missing talus. The mechanism of trauma in these cases is similar to total talar dislocations (although never clearly expounded in the various published texts!), but there may be an added element of degloving or crushing. ${ }^{3,4}$ 
Considering the mechanism of trauma and the outcomes of these injuries, it would not be wrong to hypothesize that these injuries represent a continuum. On one end, there are the low-energy subtalar dislocations that invariably have excellent outcomes, whereas on the other extreme are total talar extrusions that have high rates of complications.

So, what do we hypothesize? The injury mechanism maybe the same, with planterflexion being the common link in all of these injuries; the direction of dislocation depends upon the subsequent deforming force. The degree of dislocation also depends upon the continuation of the deforming forces, and the end result maybe a talar extrusion, through the skin, with the bone often found outside the body. The outcomes depend upon the initial force, the degree of dislocation as well as associated compounding of the injury. Orthopedic surgeons need to familiarize themselves with these relatively rare injuries, and understand the deforming mechanisms. Accurate identification of the injury, timely and anatomical reduction of the subtalar, talonavicular and/or talocrural joints and appropriate management of open wounds are the keys to successful outcomes.

\section{REFERENCES}

1. Rammelt S, Goronzy J. Subtalar dislocations. Foot Ankle Clin 2015;20(2):253-264.

2. Weston JT, Liu X, Wandtke ME, Liu J, Ebraheim NE. A systematic review of total dislocation of the talus. Orthop Surg 2015;7(2): 97-101.

3. Mohindra M, Gogna P, Thora A, Tiwari A, Singla R, Mahindra P. Early reimplantation for open total talar extrusion. J Orthop Surg (Hong Kong) 2014;22(3):304-308.

4. Smith CS, Nork SE, Sangeorzan BJ. The extruded talus: results of reimplantation. J Bone Joint Surg Am 2006;88(11):2418-2424.

Mandeep S Dhillon

MBBS, MS (Orth), FAMS, FRCS

Chairperson, AO Trauma, India

President, Indian Association for Sports Medicine

Founder President, Indian Biological Orthopedics Society

Past President, Indian Arthroplasty Association

Past President, Indian Foot and Ankle Society

Past President, North Zone IOA

Professor and Head, Department of Orthopedics

Head, Department of Physical Medicine

Postgraduate Institute of Medical Education and

Research, Chandigarh, India

e-mail: drdhillon@gmail.com

Siddhartha Sharma

Senior Resident, Department of Orthopedics

Postgraduate Institute of Medical Education and Research

Chandigarh, India 